\title{
DINASTÍNEOS (COLEOPTERA, SCARABAEIDAE, DYNASTINAE) DO PARQUE NACIONAL DO JAÚ, AMAZONAS, BRASIL
}

\author{
Ricardo ANDREAZZE'
}

RESUMO - Besouros dinastíneos foram coletados no Parque Nacional do Jaú, Estado do Amazonas, entre julho de 1993 a junho 1996, em dias de Lua minguante e nova, em 5 localidades diferentes, abrangendo principalmente a região dos baixos cursos dos rios Jaú, Carabinani e Unini. Utilizou-se para as coletas luz mista de mercúrio de $250 \mathrm{~W}$ e duas lâmpadas ultra-violeta, blacklight (BL) e black-light blue (BLB), sobre um lençol branco em periodos diários de 12 horas consecutivas. Foram identificadas 34 espécies de 14 gêneros, sendo as espécies da tribo Cyclocephalini as mais representadas (19 espécies), seguidas por Oryctini (6 spp.), Phileurini (4 spp.), Pentodontini (3 spp.) e Dynastini com 2 espécies.

Palavras-chave: Coleoptera, Scarabaeoidea, Scarabaeidae, Dynastinae, Parque Nacional do Jaú Dynastinae Beetles (Coleoptera, Scarabaeidae, Dynastinae) of Parque Nacional do Jaú, state of Amazonas, Brazil.

ABSTRACT - Dynastinae beetles were collected at the Parque Nacional do Jaú, state of Amazonas between july/1993 to june/1996, during new moon and last quarter in five different places near the lowlands of the Jau river. A mixed $250 \mathrm{~W}$ mercury vapor lamp plus two ultraviolet lights, black light (BL) and black light blue (BLB) were used for atraction on a white sheet in daily periods of 12 consecutive hours. 34 species of 14 genera were identified being the species of the tribe Cyclocephalini the more represented ( 19 species), followed by Oryctini ( $6 \mathrm{spp}$.), Phileurini (4 spp.), Pentodontini (3 spp.) and Dynastini with 2 species.

Key-words: Coleoptera, Scarabaeoidea, Scarabaeidae, Dynastinae, Parque Nacional do Jaú.

\section{INTRODUÇÃO}

Os besouros dinastíneos compreendem um grupo peculiar da família Scarabaeidae pois nela se encontram alguns dos maiores coleópteros conhecidos até hoje. Desde meados do século passado atraiu a atenção de exploradores e naturalistas nas várias regiões do mundo onde podem ser encontrados. A variedade de tamanhos, as características morfológicas que impressionam, levou a ser um dos grupos de insetos mais procurados e colecionados atualmente.

Os dinastíneos desenvolvem-se em troncos mortos de árvores lenhosas e passam a maior parte de sua vida (em média 15 meses, cerca de dois a três anos em algumas espécies) dentro destes alimentando-se da madeira onde ocorre toda a fase larval (Morón, 1985). Esses besouros ingerem grande quantidade de madeira, o que os torna eficientes decompositores, pois com suas mandibulas poderosas fragmentam partes de um tronco em espaço de tempo menor do que se ficassem somente expostos à ação das intempéries. Isso os torna importantes para a ciclagem de nutrientes dentro da manutenção do ecossistema de floresta tropical (Morón, 1987).

Segundo Endrodi (1985), a

\footnotetext{
'Instituto Nacional de Pesquisas da Amazónia (INPA), Coordenação de Pesquisas em Ecologia (CPEC), Projeto Dinâmica Biológica de Fragmentos Florestais (PDBFF), c.p. 478, 69011970 Manaus, AM, Brasil. Bolsista CNPq. e-mail: andreaze@inpa.gov.br
} 
subfamilia Dynastinae está dividida em oito tribos, sendo seis destas representadas na região Neotropical e com número de espécies muito superior às outras regiões zoogeográficas do mundo. Nos últimos anos um grande número de espécies novas têm sido descritas, havendo algo em torno de 1700 espécies já conhecidas (Endrödi, 1985; Lachaume, 1985, 1992), provavelmente metade delas ocorrendo na região Neotrópica.

A biologia dos dinastíneos também é pouco conhecida na Amazônia e para algumas espécies, as fases imaturas ainda não foram descritas. Uma vez que os adultos são coletados em sua maioria pela atração à luz a noite, conhece-se pouco sobre a atividade desses insetos, bem como as plantas em que se desenvolvem.

O colecionamento de coleópteros no Parque Nacional do Jaú, além dos subsidios que pode fornecer para o conhecimento da diversidade dos dinastineos, também pode contribuir para a expansão do conhecimento da entomologia nos trópicos. É uma área ainda pouco conhecida sob o ponto de vista entomológico, tornando-se um pré-requisito indispensável para sua preservação o estudo das espécies que ai ocorrem, seja por importantes desdobramentos tanto na área de saúde, quanto do ponto de vista estritamente biológico, taxonômico e ecológico, em face ao Plano de Manejo já implantado no Parque (Fundação Vitória Amazônica, 1998).

\section{MATERIAL E MÉTODOS}

O Parque Nacional do Jaú abrange uma área de 2.272.000ha e está localizado entre as latitudes $1^{\circ} 40^{\prime}$ a $3^{\circ} 00^{\prime}$ $\mathrm{S}$ e longitudes $61^{\circ} 25^{\prime}$ a $63^{\circ} 50^{\prime} \mathrm{W}$, delimitado pelos rios Negro, Jaú e Carabinani, no Município de Novo Airão e pelo rio Unini, no Municipio de Barcelos, no Estado do Amazonas. Toda a área do parque é de vegetação primária, com pouca interferência humana (Fundação Vitória Amazônica, 1998).

Foram realizadas coletas em cinco localidades principais (1-igarapé Miratuca; 2-Castanho; 3-rio Carabinani; 4-Miriti e 5-rio Unini) e adjacências, durante os anos de 1993 a 1996, variando-se a permanência em cada ponto de 1 a 20 dias aproximadamente (Tab.1). As localidades escolhidas tinham sido em sua maioria, pré-habitadas por moradores, estando abandonadas temporária ou definitivamente.

O método de coleta utilizado foi o mesmo empregado em levantamentos anteriores de coleópteros dinastíneos na Amazônia, que por enquanto é o mais indicado para a captura desses coleópteros (Andreazze \& Fonseca, 1998).

As coletas foram em noites consecutivas em intervalos de 12 horas seguidas (das 18:00 às 06:00) antes (Lua minguante) e durante o periodo de Lua nova, periodo esse em que há menos interferência da luz lunar.

As identificações foram feitas usando-se chaves especificas e por análise de genitália dos machos segundo Endrodi (1985) e Lachaume $(1985,1992)$ e a classificação dos Dynastinae segue a 
Tabela 1. Locais de coleta amostrados, datas, número de noites e de horas empregadas no estudo do Parque Nacional do Jaú, Amazonas, Brasil.

\begin{tabular}{|c|c|c|c|c|}
\hline Locais & Coordenadas geográficas & Datas & Noites & Horas \\
\hline \multirow[t]{3}{*}{ 1-igarapé Miratuca } & $1^{9} 57^{\circ} 08^{\prime \prime S} / 61^{\circ} 49^{\prime} 19^{\circ} \mathrm{W}$ & $14-29 / 07 / 1993$ & 15 & 180 \\
\hline & & $09-10 / 06 / 1994$ & 1 & 12 \\
\hline & & $23-29 / 07 / 1995$ & 6 & 72 \\
\hline \multirow[t]{2}{*}{ 2-Castanho, baixo rio Jaú } & $1^{\circ} 56^{\prime} \mathrm{S} / 61^{\circ} 44^{\prime} \mathrm{W}$ & $10-30 / 10 / 1993^{*}$ & 18 & 216 \\
\hline & & $22-23 / 07 / 1995$ & 1 & 12 \\
\hline \multirow[t]{3}{*}{ 3-rio Carabinani } & $1^{\circ} 58^{\prime} \mathrm{S} / 61^{\circ} 44^{\prime} \mathrm{W}$ & $06-17 / 04 / 1994$ & 11 & $\overline{132}$ \\
\hline & & $26 / 04-02 / 05 / 1995$ & 6 & 72 \\
\hline & & $29 / 7-02 / 08 / 1995$ & 4 & 48 \\
\hline 4-Miriti & $2^{2} 17^{\prime} \mathrm{S} / 62^{2} 28^{\prime} \mathrm{W}$ & $03-09 / 06 / 1994$ & 6 & 72 \\
\hline \multirow[t]{5}{*}{ 5-rio Unini } & $1^{2} 38^{\prime} 28^{\prime \prime S} / 61^{\circ} 46^{\prime} 20^{\prime \prime} \mathrm{W}$ & $20-21 / 11 / 1995$ & 1 & $\overline{12}$ \\
\hline & & $21-24 / 11 / 1995$ & 3 & 36 \\
\hline & & $11-12 / 6 / 1996$ & 1 & 12 \\
\hline & & $12-19 / 6 / 1996$ & 7 & 84 \\
\hline & & $19-25 / 6 / 1996$ & 6 & 72 \\
\hline Total & & & 86 & 1032 \\
\hline
\end{tabular}

- Em 2 noites nâo houve coleta devido a pane no motor

nomenclatura de Lawrence \& Newton (1995). O material está depositado na Coleção Entomológica do INPA.

\section{RESULTADOS E DISCUSSÃO}

Foram colecionados 2380 exemplares de 34 espécies em 14 gêneros, em 86 noites, 1032 horas de coletas (Tab.2). A tribo Cyclocephalini foi a mais representativa com 19 espécies de 5 gêneros (1974 individuos), seguida por Oryctini com 6 espécies de 4 gêneros (180 indivíduos). Do total, 13 espécies já tinham sido assinaladas anteriormente com ocorrência na região de Itacoatiara (Andreazze \& Fonseca, 1998).

Harposceles paradoxus é monotípica $e$ estava registrada apenas para a Guiana Francesa e Equador, sendo aqui assinalada pela primeira vez para o Amazonas (Endrödi, 1985; Lachaume, 1992). Esta espécie provavelmente ocorre em áreas ainda intactas, ou com pouca alteração, podendo ser bioindicadora.

As regiões próximas às desembocaduras dos rios Unini, Jaú e Carabinani, possuem maior ocupação humana do parque, porém em pequena escala, visto a ser a agricultura de subsistência praticada, em contraste à região do municipio de Itacoatiara, que possui áreas já alteradas e em constante mudança pela atividade econômica.

\section{A tribo Cyclocephalini inclui} aproximadamente 300 espécies, sendo Cyclocephala Latreille 1829 o gênero mais comum com aproximadamente 270 espécies conhecidas. Os adultos das espécies deste gênero alimentamse de pólen e outras partes de várias flores, sendo um grupo importante de polinizadores de várias espécies de palmeiras e anonáceas (Küchmeister $e t$ al., 1998). Neste trabalho, as duas espécies mais numerosas em todo o 
levantamento realizado foram: Stenocrates ligneus (1106 indivíduos) e Stenocrates carbunculus (393 inds.), ambas com distribuição geográfica na região amazônica.
A
composição
da coleopterofauna do Parque mostrou-se diferente em relação à Itacoatiara, sendo do total coletado 13 espécies comuns àquela região.

Observando-se o número de espécies obtidas em cada localidade, nota-se que, excetuando-se a região do Miriti (4), onde houve apenas 6 noites de coleta, todas as outras áreas, o número de espécies foi próximo em relação ao esforço de coleta empregado.

\section{AGRADECIMENTOS}

\section{À Fundação Vitória Amazônica} e ao INPA pelo apoio logístico e

Tabela 2. Espécies de Dynastinae coletadas no período de julho/1993 a junho/1996, no Parque Nacional do Jaú, Estado do Amazonas, Brasil. Legenda: 1-igarapé Miratuca;2-Castanho;3-rio Carabinani;4-Miriti;5-rio Unini.

\begin{tabular}{|c|c|c|c|c|c|c|c|c|c|}
\hline Tribe & Espécie & & Distribuiçăo Geográfica & 1 & 2 & 3 & 4 & 5 & Total \\
\hline Cyclocephalini & Cyclocephala bicolor Castelnau, 1840 & $\circ$ & Su,GF,Pe, Br (PA,AM,MT) & 2 & 186 & 1 & - & 2 & 191 \\
\hline 1974 indvs. & Cyclocephala colasi Endrodi, 1964 & $\circ$ & $\operatorname{Br}(A M)$ & 2 & 56 & - & - & 12 & 70 \\
\hline \multirow[t]{17}{*}{$19 \mathrm{spp}$} & Cyclocephala guianae Endrodi, 1969 & & $\mathrm{GF}, \mathrm{Br}(\mathrm{AM})$ & - & $\cdots$ & 2 & - & - & 2 \\
\hline & Cyciocephala hardyi Endrodi, 1975 & a & $\mathrm{Br}(\mathrm{A}$ & - & 1 & - & . & 3 & 4 \\
\hline & Cyciocephala parafiora Martinez, 1978 & & Gu,Br (AM) & 4 & - & - & 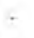 & - & 4 \\
\hline & Cyciocephaia testacea Burmeister, 1847 & $\therefore$ & EU-Ar, $\mathrm{Br}(\mathrm{AM})$ & 7 & 62 & 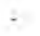 & - & 2 & 71 \\
\hline & Cyclocephala verticalis Burmeister, 1847 & $\propto$ & $\mathrm{GF}, \mathrm{Eq}, \mathrm{Pe}, \mathrm{Bo}, \mathrm{Br}$ (PA,AM,MT,SC) & - & 3 & - & - & . & 3 \\
\hline & Cyclocephala sp.1 & & $\mathrm{Br}(\mathrm{AM})$ & 4 & - & $\cdot$ & - & 8 & 12 \\
\hline & Cyclocephala sp.2 & & $\operatorname{Br}(\mathrm{AM})$ & $\rightarrow$ & 1 & $\cdot$ & - & - & 1 \\
\hline & Cyclocephala sp. 3 & & $\operatorname{Br}(A M)$ & 2 & , & $\cdot$ & - & $\cdot$ & 2 \\
\hline & Cyclocesphala sp.4 & & $\mathrm{Br}(\mathrm{AM})$ & ;- & $\rightarrow$ & 1 & + & - & 1 \\
\hline & Dysainetus dubius (Olivier, 1789) & $\circ$ & $\mathrm{Bo}, \mathrm{Br}(\mathrm{AM})$ & & - & - & - & 4 & 1 \\
\hline & Harposceles paradoxus Burmeister, 1847 & & GF,Eq, $\mathrm{Br}(\mathrm{AM})$ & - & $=$ & 3 & - & - & 3 \\
\hline & Stenocrates carbuncuilus Prell, 1938 & a & $\mathrm{Br}(\mathrm{AM})$ & 27 & 355 & 8 & 3 & - & 393 \\
\hline & Stenocrates tigneus Amow. 1911 & a & $\mathrm{Pa}, \mathrm{Br}(\mathrm{PA}, \mathrm{AM})$ & - & 664 & $\cdot$ & - & 442 & 1106 \\
\hline & Stenocrates sp. 1 & & $\operatorname{Br}(A M)$ & - & 6 & - & - & 64 & 70 \\
\hline & Stenocrates sp. 2 & & $\mathrm{Br}(\mathrm{AM})$ & $\cdot$ & - & $\cdot$ & 3 & 26 & 29 \\
\hline & Surufu fenni Racliffe, 1981 & & $\mathrm{Br}(\mathrm{AM})$ & $\cdot$ & 3 & - & - & : & 3 \\
\hline & Surutu seabrai Andretta et Martinez 1956 & & $\mathrm{Br}(\mathrm{PA}, \mathrm{AM})$ & 2 & 2 & 3 & 1 & - & 8 \\
\hline Pertodontini & Ligyrus ebenus (De Geer, 1774) & 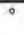 & $\mathrm{AC}, \mathrm{Co}, \mathrm{Ve}, \mathrm{Tr}, \mathrm{Gu}, \mathrm{Pe}, \mathrm{Bo}, \mathrm{Br}(\mathrm{AM})$ & 2 & 80 & 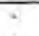 & - & - & 82 \\
\hline 206 indvs. & Ligyns sp. 1 & & $\operatorname{Br}(A M)$ & - & - & 7 & - & 8 & 9 \\
\hline 3 spp. & Oryligyrus zoilus (Olivier, 1789) & ? & $\mathrm{GF}, \mathrm{Co}, \mathrm{BO}, \mathrm{Br}$ (AM,PA,SP) & 18 & 1 & - & - & 96 & 715 \\
\hline Oryctini & Coelosis biloba (Linnaeus, 1767) & 0 & $A C \cdot A r, B r(A M)$ & 1 & $\cdot$ & 2 & - & $\cdot$ & 3 \\
\hline 180 indvs. & Enema pan (Fabricius, 1775) & & $\mathrm{GF}, \mathrm{Co}, \mathrm{Eq}, \mathrm{Pe}, \mathrm{Bo}, \mathrm{Pa}, \mathrm{Br}$ (AM) & - & 93 & 3 & - & 69 & 165 \\
\hline \multirow[t]{4}{*}{$6 \mathrm{spp}}$. & Megaceras lycaon Endrodi, 1975 & & GF, $\mathrm{Pe}, \mathrm{Br}(\mathrm{AM})$ & - & - & 1 & 5 & 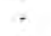 & 6 \\
\hline & Strategus aloeus (Linnaeus, 1758) & $\because$ & $\mathrm{EU}-\mathrm{Ar}, \mathrm{Br}(\mathrm{AM})$ & 2 & 1 & - & , & $\cdot$ & 3 \\
\hline & Strategus sp. 1 & & $\mathrm{Br}(\mathrm{AM})$ & $\cdot$ & 1 & 1 & 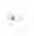 & $\cdot$ & 2 \\
\hline & Strategus sp. 2 & & $\mathrm{Br}(\mathrm{AM})$ & - & $\cdot$ & 1 & 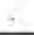 & - & 1 \\
\hline Dynastini & Megasoma actaeon (Limaeus, 1758) & 0 & $\mathrm{Co}, \mathrm{Eq}, \mathrm{Bo}, \mathrm{Gu}, \mathrm{Ve}, \mathrm{Br}(\mathrm{AM})$ & 13 & . & 3 & + & 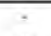 & 16 \\
\hline 18 indvs./ 2 spp. & Megasoma mars (Reiche, 1852) & & $\mathrm{Co}, \mathrm{Br}(\mathrm{AM})$ & . & 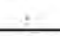 & $\stackrel{-}{-}$ & + & 2 & 2 \\
\hline Phileusini & Homophileunus sp 1 & & $\mathrm{Br}(\mathrm{AM})$ & - & - & 1 & $\cdot$ & . & 1 \\
\hline 7 indvs. & Homophileurus sp 2 & & $\mathrm{Br}(\mathrm{AM})$ & 1 & - & - & $\cdot$ & - & 1 \\
\hline \multirow[t]{2}{*}{4 spp. } & Phrileunus didymus Linnaeus, 1758 & & $\mathrm{AC}-\mathrm{Pa}$ & 1 & - & 1 & - & - & 2 \\
\hline & Phileurus sp.1 & & $\operatorname{Br}(\mathrm{AM})$ & + & 1 & $T$ & - & 1 & 3 \\
\hline Total de individuos & & & & 87 & 1515 & 31 & 12 & 785 & 2380 \\
\hline Total de especies & & & & 15 & 17 & 16 & 4 & 14 & 34 \\
\hline Horas de coleta & & & & 264 & 229 & 252 & 72 & 216 & 1032 \\
\hline
\end{tabular}

$\mathrm{EU}=$ Estados Unidos; $\mathrm{AC}=$ América Central; $\mathrm{GF}=\mathrm{Guiana}$ Francesa; Su=Suriname; Gu=Guiana; $\mathrm{A} 23$ Tr-Trinidad; $\mathrm{Ve}=$ Venezuela; $\mathrm{Co}=$ Colombia; $\mathrm{Pe}=\mathrm{Peru} ; \mathrm{Eq}=$ Equador; $\mathrm{Bo}=\mathrm{Bolivia} ; \mathrm{Pa}=\mathrm{Paraguai} ; \mathrm{Ar}=\mathrm{Argentina}$.

$\left({ }^{\circ}\right)=$ espécies assinaladas em Itacoatiara (Andreazze \& Fonseca, 1998) 
condições para a execução deste trabalho. À pesquisadora Catarina da Silva Motta (INPA/CPEN) pelo incentivo, auxilio e coleta de material em excursões que não participei.

\section{Bibliografia citada}

Andreazze, R. \& Fonseca, C.R.V. 1998. Dinastineos (Coleoptera, Scarabaeoidea, Melolonthidae) em uma área de terra firme na Amazônia Central, Brasil. Acta Amazonica 28(1): 59-66.

Endrödi, S. 1985 The Dynastinae of the world. Dr. W. Junk Publishers, Budapest, Hungary, 800 p. 56 plates.

Fundação Vitória Amazônica. 1998. A Gênese de um Plano de Manejo. O caso do Parque Nacional do Jaú. Fundação Vitória Amazônica, Manaus, FVA 113pp.

Küchmeister, H.; Webber, A.C.; SilberbauerGottsberger, I.; Gottsberger, G. 1998. A polinização e sua relação com a termogênese em espécies de Arecaceae e Annonaceae da Amazônia Central. Acta Amazonica. 28(3): 217-245.

Lachaume,G. 1985. Les Coléoptères du Monde. The Beetles of the World. Vol. 5 Dynastini 1 (première partie). Sciences Nat. Venette. France 85 pp, 29 pls.

Lachaume,G. 1992. Les Coléoptères du Monde. The Beetles of the World. Vol.14 Dynastidae américains. Sciences Nat. Venette. France 89 pp, 13 pls.

Lawrence, J. F. and A. F. Newton, Jr. 1995. Families and subfamilies of Coleoptera (with selected genera, notes, references and data on family-group names). pp. 7791006 in: J. Pakaluk and S.A. Slipinski (eds.): Biology, Phylogeny, and Classifcation of Coleoptera: Papers Celebrating the 80th Birthday of Roy A. Crowson. Museum i Instytut Zoologii PAN, Warszawa.
Morón, M.A. 1985. Los insectos degradadores, un factor poco estudiado en los bosques de Mexico. Folia Entomológica Mexicana 65: 131-137.

Morón, M.A. 1987. Los estados inmaduros de Dynastes hyllus Chevrolat (Coleoptera: Melolonthidae: Dynastinae); con observaciones sobre su biologia y el crecimiento alometrico del imago. Folia Entomológica Mexicana 72: 33-74. 